\title{
Some Properties of the Size-Biased Janardan Distribution
}

\author{
Shakila Bashir*, Mujahid Rasul \\ Department of Statistics, Forman Christian College a Chartered University, Lahore, Pakistan \\ Email address: \\ shakilabashir@fccollege.edu.pk (S. Bashir),mujahidrasul@fccollege.edu.pk (M. Rasul) \\ ${ }^{*}$ Corresponding author
}

\section{To cite this article:}

Shakila Bashir, Mujahid Rasul. Some Properties of the Size-Biased Janardan Distribution. American Journal of Theoretical and Applied Statistics. Vol. 5, No. 5, 2016, pp. 305-310. doi: 10.11648/j.ajtas.20160505.19

Received: July 3, 2016; Accepted: July 19, 2016; Published: September 21, 2016

\begin{abstract}
Janardan Distribution is one of the important distributions from lifetime models and it has many applications in real life data. A size-biased form of the two parameter Janardan distribution has been introduced in this paper, of which the size-biased Lindley distribution is a special case. Its moments, median, skewness, kurtosis and Fisher index of dispersion are derived and compared with the size-biased Lindley distribution. The shape of the size-biased Janardan distribution is also discussed with graphs. The survival function and hazard rate of the size-biased Janardan distribution have been derived and it is concluded that the hazard rate of the distribution is monotonically increasing. The flexibility in the reliability measures of the size-biased Janardan distribution have been discussed by stochastic ordering. To estimate the parameters of the size-biased Janardan distribution maximum likelihood equations are developed.
\end{abstract}

Keywords: Size-Biased Distributions, LD, JD, PJD, SBLD, SBJD, MLE, Stochastic Ordering, IFR

\section{Introduction}

Size-biased distributions are the special cases of the weighted distributions. [6] introduced the weighted distributions to model ascertainment bias and later was discussed by [13]. [11] \& [12] discussed the applications of weighted distributions and size biased sampling in real life. These distributions arise in practice when observations from a sample are recorded with unequal probability and provide a unifying approach for the problems where the observations fall in the non-experimental, non-replicate, and non-random categories. If the random variable $\mathrm{X}$ has the probability distributions function (pdf), $f(x ; \theta)$ then the size-biased distribution is of the form

$$
f(x ; \theta)=\frac{x^{m} f_{0}(x ; \theta)}{\mu_{m}^{\prime}}
$$

Where $\mu_{m}^{\prime}=\int_{0}^{\infty} x^{m} f_{0}(x ; \theta) d x$ for $\alpha=1 \& \alpha=2$ we get the size-biased and area-biased distributions respectively. [3] proposed a weighted Lindley distribution by using a new weight function. Various properties of the model have been derived and the shape of the hazard rate is also discussed. [1] derived size-biased gamma distribution (SBGMD). They derived the characterizing properties of the SBGMD including Shannon entropy and Fisher's information matrix. They also derived Baye's estimator of the SBGMD using different priors. [5] examined the size-biased versions of the generalized beta of the first kind, generalized beta of the second kind and generalized gamma distributions. They discussed broader applications of the size-biased distributions in forestry sampling, modeling and analysis. [2] derived sizebiased Pareto distribution and discussed upper record values of the size-biased Pareto distribution. They proposed some recurrence relations satisfied by the single and product moments of upper record values form size-biased Pareto distribution.

[17] derived size-biased Poisson Lindley distribution (SBPLD) and its moments. They estimated parameter of the SBPLD and apply the model on thunderstorms. They concluded that the size-biased Poisson Lindley distribution (SBPLD) gives much closer fit than the sizebiased Poisson distribution (SBPD). [10] derived some size-biased probability distributions and their generalizations. These distributions provide a unifying approach for the problems where the observations fall in the non-experimental, non- replicated, and nonrandom 
categories.

[9] introduced one parameter Lindley distribution (LD) as

$$
f(x ; \theta)=\frac{\theta^{2}}{(\theta+1)}(1+x) e^{-\theta x}, \quad x>0 \quad \theta>0
$$

[8] discussed various properties of the Lindley distribution and showed that Lindley distributions provide a better fit than exponential distributions. [7] introduced the size-biased Poisson Lindley distribution considering the size-biased form of the mixture of Poisson Lindley distribution. They developed various properties of the sizebiased Lindley distribution and its applications on biological data. [16] introduced a two parameter continuous distribution named as Janardan distribution (JD) and derived its various properties including moments, failure rate function, mean residual life function and stochastic ordering. They also discussed the estimation methods for JD and apply it on waiting time data. The probability density function of the JD is

$$
f(x ; \theta, \alpha)=\frac{\theta^{2}}{\alpha\left(\theta+\alpha^{2}\right)}(1+\alpha x) e^{-\frac{\theta}{\alpha} x}, \quad x>0, \quad \theta>0, \alpha>0 .
$$

It can be seen that for $\alpha=1$, the LD (2) is a special case of JD (3). The JD is a mixture of exponential $\left(\frac{\theta}{\alpha}\right)$ and Gamma $\left(2, \frac{\theta}{\alpha}\right)$ distribution. The mean of the JD is

$$
\mu_{1}^{\prime}=\frac{\alpha\left(\theta+2 \alpha^{2}\right)}{\theta\left(\theta+\alpha^{2}\right)}
$$

[15] introduced the mixture of Poisson and Janardan distribution named discrete Poisson-Janardan distribution (PJD). They developed properties and parameter estimation of the PJD and applied it on two data sets, distribution of mistakes in copying groups of random digits and distribution of Pyrausta nublilalis.[4] derived Poisson area-biased Lindley distribution including its structural properties. The applications of the Poisson area-biased Lindley distribution are discussed in biostatistics.

In this paper the size-biased form of the Janardan distribution of which size-biased Lindley distribution is a special case, has been suggested and various properties of size-biased Janardan distribution (SBJD) comparing with size-biased Lindley Distribution.

\section{The Size-Biased Janardan Distribution (SBJD)}

By using equation (1) the probability density function of the size-biased Janardan distribution (SBJD) is

$$
f(x ; \theta, \alpha)=\frac{\theta^{3}}{\alpha^{2}\left(\theta+2 \alpha^{2}\right)} x(1+\alpha x) e^{-\frac{\theta}{\alpha} x}, \quad x>0, \quad \theta>0, \alpha>0 .
$$

It is observed that for $\alpha=1$, the SBJD (5) approaches to size-biased Lindley distribution (SBLD) with probability density function

$$
f(x ; \theta)=\frac{\theta^{3}}{(\theta+2)} x(1+x) e^{-\theta x}, \quad x>0, \quad \theta>0 .
$$

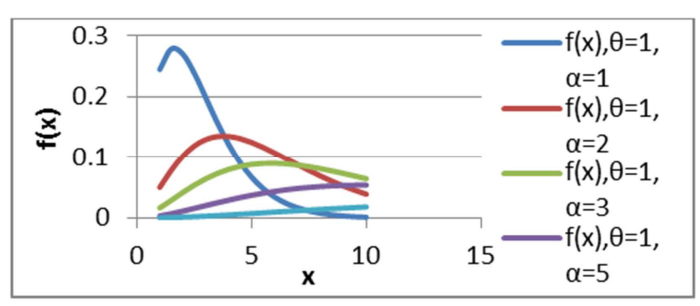

Fig. 1. The pdf graph for SBJD for $\theta=1$ and $\alpha=1,2,3,5,10$.

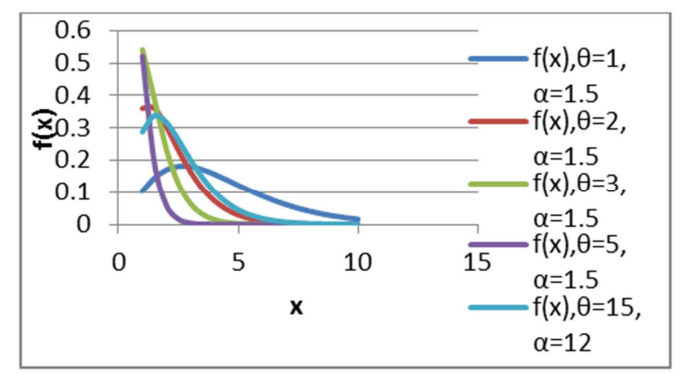

Fig. 2. The pdf graph for SBJD for different values of parameters.

From Fig. $1 \& 2$ it can be seen that the size-biased Janardan distribution is positively skewed. In Fig. 1. With $\theta=1$, as we increase the value of $\alpha$ the shape of the model is going to flatting and for lower value of $\alpha$ the model is peaked. In Fig. 2. with $\alpha=1.5 \& \theta=2,3$ the shape of the model is peaked. For $\alpha=1.5 \& \theta=1$, and $\alpha=12 \& \theta=15$, the shape of the model is nearly similar to normal.

The cumulative distribution function (cdf) of the SBJD (5) is

$$
F(x)=1-\frac{\alpha\left(\theta+2 \alpha^{2}\right)+\theta x\left(\theta+2 \alpha^{2}\right)+\theta^{2} \alpha x}{\alpha\left(\theta+2 \alpha^{2}\right)} e^{-\frac{\theta}{\alpha} x}, x>0, \theta>0, \alpha>0 .
$$

Some basic measures (moments, skewness and kurtosis) of the SBJD (5) and SBLD (6), are given in the following table

Table 1. Some measures of the SBJD and SBLD.

\begin{tabular}{lll}
\hline Measure & SBJD & SBLD for $\alpha=1$ in SBJD \\
\hline$\mu_{1}^{\prime}=$ & $\frac{2 \alpha\left(\theta+3 \alpha^{2}\right)}{\theta\left(\theta+2 \alpha^{2}\right)}$ & $\frac{2(\theta+3)}{\theta(\theta+2)}$ \\
\hline
\end{tabular}




\begin{tabular}{lll}
\hline Measure & SBJD & SBLD for $\alpha=1$ in SBJD \\
\hline$\mu_{2}^{\prime}=$ & $\frac{6 \alpha^{2}\left(\theta+4 \alpha^{2}\right)}{\theta^{2}\left(\theta+2 \alpha^{2}\right)}$ & $\frac{6(\theta+4)}{\theta^{2}(\theta+2)}$ \\
$\mu_{3}^{\prime}=$ & $\frac{24 \alpha^{3}\left(\theta+5 \alpha^{2}\right)}{\theta^{3}\left(\theta+2 \alpha^{2}\right)}$ & $\frac{24(\theta+5)}{\theta^{3}(\theta+2)}$ \\
$\mu_{4}^{\prime}=$ & $\frac{120 \alpha^{4}\left(\theta+6 \alpha^{2}\right)}{\theta^{4}\left(\theta+2 \alpha^{2}\right)}$ & $\frac{120(\theta+6)}{\theta^{4}(\theta+2)}$ \\
$\mu_{2}=$ & $\frac{2 \alpha^{2}\left(\theta^{2}+6 \theta \alpha^{2}+6 \alpha^{4}\right)}{\theta^{2}\left(\theta+2 \alpha^{2}\right)^{2}}$ & $\frac{2\left(\theta^{2}+6 \theta+6\right)}{\theta^{2}(\theta+2)^{2}}$ \\
$\mu_{3}=$ & $\frac{4 \alpha^{3}\left(\theta^{3}+9 \theta^{2} \alpha^{2}+18 \theta \alpha^{4}+12 \alpha^{6}\right)}{\theta^{3}\left(\theta+2 \alpha^{2}\right)^{3}}$ & $\frac{4\left(\theta^{3}+9 \theta^{2}+18 \theta+12\right)}{\theta^{3}(\theta+2)^{3}}$ \\
$\mu_{4}=$ & $\frac{24 \alpha^{4}\left(\theta^{4}+12 \theta^{3} \alpha^{2}+42 \theta^{2} \alpha^{4}+212 \theta \alpha^{6}+30 \alpha^{8}\right)}{\theta^{4}\left(\theta+2 \alpha^{2}\right)^{4}}$ & $\frac{24\left(\theta^{4}+12 \theta^{3}+42 \theta^{2}+212 \theta+30\right)}{\theta^{4}(\theta+2)^{4}}$ \\
$\gamma_{1}=$ & $\frac{\sqrt{2}\left(\theta^{3}+9 \theta^{2} \alpha^{2}+18 \theta \alpha^{4}+12 \alpha^{6}\right)}{\left(\theta^{2}+6 \theta \alpha^{2}+6 \alpha^{4}\right)^{3 / 2}}$ & $\frac{\sqrt{2}\left(\theta^{3}+9 \theta^{2}+18 \theta+12\right)}{\left(\theta^{2}+6 \theta+6\right)^{3 / 2}}$ \\
\hline$\beta_{2}=$ & $\frac{6\left(\theta^{4}+12 \theta^{3} \alpha^{2}+42 \theta^{2} \alpha^{4}+212 \theta \alpha^{6}+30 \alpha^{8}\right)}{\left(\theta^{2}+6 \theta \alpha^{2}+6 \alpha^{4}\right)^{2}}$ & $\frac{6\left(\theta^{4}+12 \theta^{3}+42 \theta^{2}+212 \theta+30\right)}{\left(\theta^{2}+6 \theta+6\right)^{2}}$ \\
\hline
\end{tabular}

It can be seen that for both the SBJD and SBLD, $\left(\gamma_{1}, \beta_{2}\right) \rightarrow\left(\frac{2 \sqrt{3}}{3}, 30\right)$ as $\theta \rightarrow 0$. Therefore the SBJD and SBLD are positively skewed and leptokurtic.

The Fisher index of dispersion of the SBJD is

$$
F I(X)=\frac{\sqrt{\left(\theta^{2}+6 \theta \alpha^{2}+6 \alpha^{4}\right)}}{\sqrt{2}\left(\theta+3 \alpha^{2}\right)}
$$

For $F I(X)<=>1$, the SBJD is under dispersed, equi dispersed and over dispersed respectively.

Median of the SBJD is

$$
\text { median }=\frac{\theta}{\left(\theta+2 \alpha^{2}\right)}\left[\Gamma\left(2, \frac{\theta}{\alpha} m\right)+\frac{\alpha^{2}}{\theta} \Gamma\left(3, \frac{\theta}{\alpha} m\right)\right] \text {, }
$$

Where $\int_{0}^{x} x^{n-1} e^{-x} d x=\Gamma(n, t)$ is incomplete gamma function.

Theorem 2.1. Let $x_{1}, x_{2}, \cdots, x_{n}$ be random sample having pdf $f(x)$ from SBJD then show that

$$
\frac{d \varphi(x)}{f(x)}=\frac{x}{\mu_{1}^{\prime}}
$$

where $\varphi(x)=\frac{1}{\mu_{1}^{\prime}} \int_{0}^{x} u f(u) d u$ and $\mu_{1}^{\prime}$ is the mean of the SBJD.

Proof. By using the probability distribution function in (5) we have

$$
\begin{gathered}
\varphi(x)=\frac{\theta^{4}}{2 \alpha^{3}\left(\theta+3 \alpha^{2}\right)} \int_{0}^{x} u^{2}(1+\alpha u) e^{-\frac{\theta}{\alpha} u} d u \\
\varphi(x)=1-e^{-\frac{\theta}{\alpha} x}-\frac{\theta^{2}}{2 \alpha^{2}} x^{2} e^{-\frac{\theta}{\alpha} x}-\frac{\theta}{\alpha} x e^{-\frac{\theta}{\alpha} x}-\frac{\theta^{3}}{2 \alpha\left(\theta+3 \alpha^{2}\right)} x^{3} e^{-\frac{\theta}{\alpha} x} .
\end{gathered}
$$

Taking derivative of (11),

$$
d \varphi(x)=\frac{\theta^{4}}{2 \alpha^{3}\left(\theta+3 \alpha^{2}\right)} x^{2}(1+\alpha x) e^{-\frac{\theta}{\alpha} x} .
$$

Hence substituting the values we get,

$$
\frac{d \varphi(x)}{f(x)}=\frac{x}{2 \alpha\left(\theta+3 \alpha^{2}\right) / \theta\left(\theta+2 \alpha^{2}\right)}=\frac{x}{\mu_{1}^{\prime}} .
$$

\section{Reliability Measures of the SBJD}

The survival function of the SBJD is 


$$
S(x)=\frac{\alpha\left(\theta+2 \alpha^{2}\right)+\theta x\left(\theta+2 \alpha^{2}\right)+\theta^{2} \alpha x}{\alpha\left(\theta+2 \alpha^{2}\right)} e^{-\frac{\theta}{\alpha} x}
$$

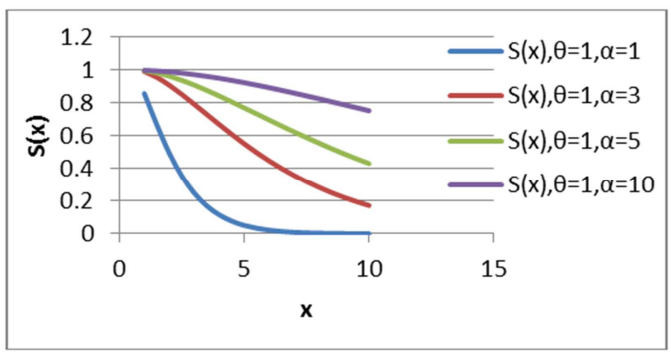

Fig. 3. Graph of the survival function for SBJD for different values of parameters.

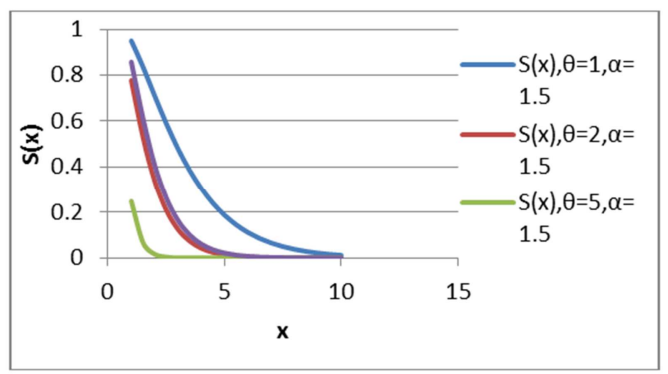

Fig. 4. Graph of the survival function for SBJD for different values of parameters.

The hazard rate function of the SBJD is

$$
h(x)=\frac{\theta^{3} x(1+\alpha x)}{\alpha\left[\alpha\left(\theta+2 \alpha^{2}\right)+\theta x\left(\theta+2 \alpha^{2}\right)+\theta^{2} \alpha x\right]}
$$

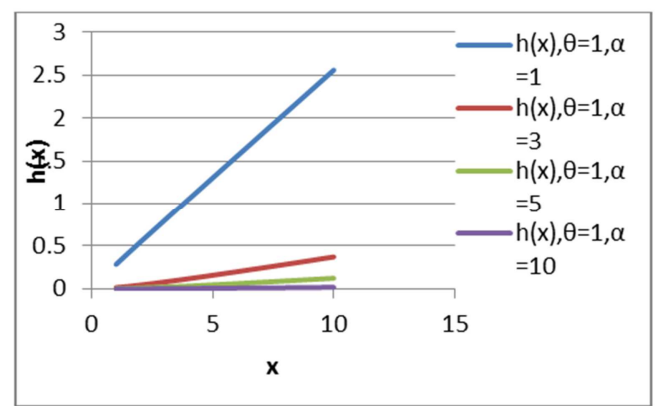

Fig. 5. Graph of the hazard function for SBJD for different values of parameters.

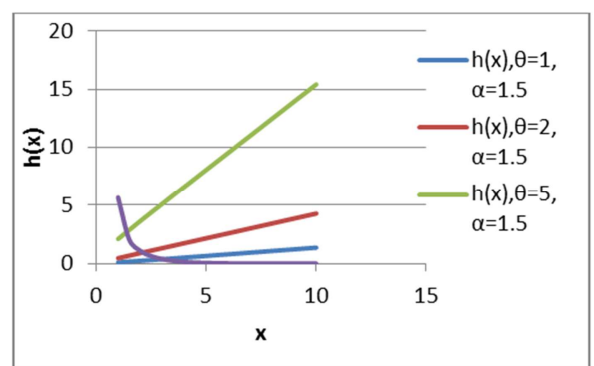

Fig. 6. Graph of the hazard function for SBJD for different values of parameters.
From fig. $3 \& 4$, it can be seen that the survival function of the SBJD is in decreasing trend and fig. $4 \& 5$, the hazard function of the SBJD is monotonically increasing. Moreover for $\alpha=1,(15)$ and (16) are the survival function and hazard function of the SBLD respectively.

Lemma 1. Let $f(x)$ is a twice differentiable density function of a continuous random variable $\mathrm{x}$ chosen from SBJD:

$$
\eta(x)=-\frac{f^{\prime}(x)}{f(x)}
$$

Then suppose the derivative of $\eta(x)$ is exist and $\eta(x)>0$ for SBJD.

$$
\text { i-e. } \eta^{\prime}(x)=\frac{1+2 \alpha x}{x^{2}(1+\alpha x)^{2}}>0 \text {; for all } x>0 \text {. }
$$

It shows that hazard function of the SBJD is monotonically increasing (IFR)

\section{Stochastic Ordering}

A random variable $\mathrm{X}$ is said to be smaller than a random variable $\mathrm{Y}$ in the

i. Stochastic order $\left(X \leq_{s t} Y\right)$ if $F_{X}(x) \geq F_{Y}(x)$ for all $\mathrm{x}$

ii. Hazard rate order $\left(X \leq_{h r} Y\right)$ if $h_{X}(x) \geq h_{Y}(x)$ for all $\mathrm{x}$

iii. Mean residual life order $\left(X \leq_{m r l} Y\right)$ if $m_{X}(x) \leq m_{Y}(x)$ for all $\mathrm{x}$

iv. Likelihood ratio order $\left(X \leq_{l r} Y\right)$ if $\frac{f_{X}(x)}{f_{Y}(x)}$ decreases in $\mathrm{x}$.

[14] considered the following results for establishing stochastic ordering of distributions

$$
\begin{aligned}
X & \leq_{l r} Y \Rightarrow X \leq_{h r} Y \Rightarrow X \leq_{m r l} Y \\
& \Downarrow \\
X & \leq_{s t} Y
\end{aligned}
$$

Theorem 3.1. Let a random variable $X$ from SBJD $\left(\theta_{1}, \alpha_{1}\right)$ and another random variable $\mathrm{Y}$ from $\operatorname{SBJD}\left(\theta_{2}, \alpha_{2}\right)$. If $\alpha_{1}=\alpha_{2}$ and $\theta_{1} \geq \theta_{2}$ (or if $\theta_{1}=\theta_{2}$ and $\alpha_{1} \leq \alpha_{2}$ ) then $X \leq_{l r} Y \Rightarrow X \leq_{h r} Y \Rightarrow X \leq_{m r l} Y \Rightarrow X \leq_{s t} Y$

Proof. Let

$$
\begin{gathered}
\frac{f\left(x ; \theta_{1}, \alpha_{1}\right)}{f\left(y ; \theta_{2} \alpha_{2}\right)}=\left(\frac{\theta_{1} \alpha_{2}}{\theta_{2} \alpha_{1}}\right)^{2}\left(\frac{\theta_{2}+2 \alpha_{2}^{2}}{\theta_{1}+2 \alpha_{1}^{2}}\right)\left(\frac{1+\alpha_{1} x}{1+\alpha_{2} x}\right) e^{-x\left(\frac{\theta_{1} \alpha_{2}-\theta_{2} \alpha_{1}}{\alpha_{1} \alpha_{2}}\right)} \\
\log \frac{f\left(x ; \theta_{1}, \alpha_{1}\right)}{f\left(y ; \theta_{2} \alpha_{2}\right)}=2 \log \left(\frac{\theta_{1} \alpha_{2}}{\theta_{2} \alpha_{1}}\right)+\log \left(\frac{\theta_{2}+2 \alpha_{2}^{2}}{\theta_{1}+2 \alpha_{1}^{2}}\right) \\
+\log \left(1+\alpha_{1} x\right)-\log \left(1+\alpha_{2} x\right)-x\left(\frac{\theta_{1} \alpha_{2}-\theta_{2} \alpha_{1}}{\alpha_{1} \alpha_{2}}\right)
\end{gathered}
$$




$$
\frac{d}{d x} \log \frac{f\left(x ; \theta_{1}, \alpha_{1}\right)}{f\left(y ; \theta_{2} \alpha_{2}\right)}=\frac{\alpha_{1}-\alpha_{2}}{\left(1+\alpha_{1} x\right)\left(1+\alpha_{2} x\right)}-\frac{\theta_{1} \alpha_{2}-\theta_{2} \alpha_{1}}{\alpha_{1} \alpha_{2}}
$$

Case (i): $\alpha_{1}=\alpha_{2}$ and $\theta_{1} \geq \theta_{2} \quad$, then $\frac{d}{d x} \log \frac{f\left(x ; \theta_{1}, \alpha_{1}\right)}{f\left(x ; \theta_{2}, \alpha_{2}\right)}<0$. It means that

$$
X \leq_{l r} Y \Rightarrow X \leq_{h r} Y \Rightarrow X \leq_{m r l} Y \Rightarrow X \leq_{s t} Y
$$

Case (i): $\alpha_{1} \leq \alpha_{2}$ and $\theta_{1}=\theta_{2}$, then $\frac{d}{d x} \log \frac{f\left(x ; \theta_{1}, \alpha_{1}\right)}{f\left(x ; \theta_{2}, \alpha_{2}\right)}<0$. It means that

$$
X \leq_{l r} Y \Rightarrow X \leq_{h r} Y \Rightarrow X \leq_{m r l} Y \Rightarrow X \leq_{s t} Y
$$

This theorem shows the flexibility of the SBJD in the context of reliability measures (stochastic ordering, hazard rate ordering, mean residual ordering and likelihood ratio ordering).

\section{Estimation of Parameters}

Maximum Likelihood Estimates (MLE): Let $x_{1}, x_{2}, \cdots, x_{n}$ be random samples from the size-biased Janardan distribution in (2.1) then the likelihood estimates function of the SBJD is

$$
L(\theta, \alpha)=\frac{\theta^{3 n}}{\alpha^{2 n}\left(\theta+2 \alpha^{2}\right)^{n}} e^{-\frac{\theta}{\alpha} \sum_{i=1}^{n} x_{i}} \prod_{i=1}^{n} x_{i}\left(1+\alpha x_{i}\right)
$$

The two $\log$ likelihood equations for $\theta \& \alpha$ are

$$
\begin{gathered}
\frac{\partial \log L(\theta, \alpha)}{\partial \theta}=\frac{3 n}{\theta}-\frac{n}{\theta+2 \alpha^{2}}-\frac{\sum_{i=1}^{n} x_{i}}{\alpha}=0 \\
\frac{\partial \log L(\theta, \alpha)}{\partial \alpha}=\frac{\theta}{\alpha^{2}} \sum_{i=1}^{n} x_{i}+\sum_{i=1}^{n} \frac{x_{i}}{1+\alpha x_{i}}-\frac{2 n}{\alpha}-\frac{4 n \alpha}{\theta+2 \alpha^{2}}=0
\end{gathered}
$$

The equations (23) and (24) cannot be solved directly. However in order to solve these equations we derive the derivatives $\frac{\partial^{2} \log L(\theta, \alpha)}{\partial \theta^{2}}, \frac{\partial^{2} \log L(\theta, \alpha)}{\partial \alpha^{2}}, \frac{\partial^{2} \log L(\theta, \alpha)}{\partial \theta \partial \alpha}$ for extreme conditions respect to two variables:

$$
\begin{gathered}
\frac{\partial^{2} \log L(\theta, \alpha)}{\partial \theta^{2}}=-\frac{3 n}{\theta^{2}}+\frac{n}{\left(\theta+2 \alpha^{2}\right)^{2}} \\
\frac{\partial^{2} \log L(\theta, \alpha)}{\partial \alpha^{2}}=\frac{2 n}{\alpha^{2}}-\frac{2 \theta n \bar{x}}{\alpha^{3}}-\sum_{i=1}^{n} \frac{x_{i}^{2}}{\left(1+\alpha x_{i}\right)^{2}}+\frac{4 n}{\theta+2 \alpha^{2}}
\end{gathered}
$$

$$
\frac{\partial^{2} \log L(\theta, \alpha)}{\partial \theta \partial \alpha}=\frac{4 n \alpha}{\left(\theta+2 \alpha^{2}\right)^{2}}+\frac{n \bar{x}}{\alpha^{2}}
$$

Hence, (by the formula) we obtain that

$$
\left[\begin{array}{ll}
\frac{\partial^{2} \log L(\theta, \alpha)}{\partial \theta^{2}} & \frac{\partial \log L(\theta, \alpha)}{\partial \theta \partial \alpha} \\
\frac{\partial \log L(\theta, \alpha)}{\partial \theta \partial \alpha} & \frac{\partial \log L(\theta, \alpha)}{\partial \alpha^{2}}
\end{array}\right]_{\substack{\hat{\theta}=\theta_{0} \\
\hat{\alpha}=\alpha_{0}}}\left[\begin{array}{l}
\hat{\theta}-\theta_{0} \\
\hat{\alpha}-\alpha_{0}
\end{array}\right]=\left[\begin{array}{l}
\frac{\partial \log L(\theta, \alpha)}{\partial \theta} \\
\frac{\partial \log L(\theta, \alpha)}{\partial \alpha}
\end{array}\right]_{\substack{\hat{\theta}=\theta_{0} \\
\hat{\alpha}=\alpha_{0}}}
$$

These equations can be solved iteratively till sufficiently close estimates of $\theta \& \alpha$ are obtained.

\section{Conclusion}

As we know that the Janardan Distribution has wide applicationsin lifetime models. A size-biased form of the two parameter Janardan distribution is derived in this paper and it has been noted that it is a special case of the size-biased Lindley distribution. Moments, median, skewness, kurtosis and Fisher index of dispersion of the size-biased Janardan distributionare derived and compared with the size-biased Lindley distribution. From the graphs of theprobability distribution function of the derived model it can be seen that the shape of the size-biased Janardan distribution is positively skewed. The survival function and hazard rate of the size-biased Janardan distribution have been derived. From graphs and lemma 1 it is concluded that the hazard rate of the distribution is monotonically increasing (IFR). The flexibility in the reliability measures of the size-biased Janardan distribution have been discussed by stochastic ordering. Maximum likelihood equations are developedto estimate the parameters of the size-biased Janardan distribution. The parameters of the size-biased JD can be estimated by simulations.

\section{References}

[1] Ahmed, A., Reshi, J. A. and Mir, K. A (2013). Structural properties of size-biased Gamma distribution. IOSR Journal of Mathematics, 5 (2), 55-61.

[2] Bashir, S. and Ahmad, M. (2015). Recurrence relations for single and product moments of record values from size-biased Pareto distribution. International Journal of Economic and Business Review, 3 (5), 139-144.

[3] Bashir, S. and Rasul, M. (2015). Some properties of the weighted Lindley distribution. International Journal of Economic and Business Review, 3 (8), 11-17.

[4] Bashir, S. and Rasul, M. (2016). Poisson area-biased Lindley distribution and its applications in biostatistics. Biometrics \& Biostatistics International Journal, 3 (1), 1-10.

[5] Ducey, M. J. and Gove, J. H. (2014). Size-biased distributions in the generalized beta family, with applications to forestry. An International Journal of Forest Research, 88 (1), 143-151. 
[6] Fisher, R. A. (1934). The effects of methods of ascertainment upon the estimation of frequencies. Ann. Eugenics, 6, 13-25.

[7] Ghitany, M. E. and Al-Mutairi, D. (2008). Size-biased Poisson Lindley distribution and its applications. METRON, LXVI, $\mathrm{n}$. 3, 299-311.

[8] Ghitany, M. E., Atieh, B., and Nadarajah, S. (2008). Lindley distribution and its applications, Mathematics and Computers in Simulation, 78 (4), 493-506.

[9] Lindley, D, V. (1958). Fiducial distributions and Bayes theorem. Journal of Royal Statistical Society, 20, 102-107.

[10] Mir, K. A. and Ahmad, M. (2009). Size-biased distributions and their applications. Pakistan Journal of Statistics, 25 (3), 283-294.

[11] Patil, G. P. and Rao, C. R. (1977). Weighted distributions: a survey of their applications, In P. R. Krishnaiah, (Eds.), Applications of statistics (pp. 383-405). Amsterdam, NorthHolland.

[12] Patil, G. P. and Rao, C. R. (1978). Weighted distributions and size-biased sampling with applications to wildlife populations and human families, Biometrics, 34, 179-189.

[13] Rao, C. R. (1965). On discrete distributions arising out of ascertainment, In G. P. Patil (Eds.), Classical and contagious discrete distributions (pp. 320-332). Calcutta, Pergamon Press and Statistical Publishing Society.

[14] Shaked, M., Shanthikumar, J. G. and Valdez-Torres, J. B. (1994). Discrete Probabilistic Orderings in Reliability Theory. StatisticaSinica, 4, 567-579.

[15] Shanker, et al. (2014). The discrete Poisson Janardan distribution with applications. International Journal of Soft Computing and Engineering, 4 (2), 31-33.

[16] Shanker, R. et al (2013). Janardan distribution and its applications to waiting time data. Indian Journal of Applied Research, 3 (8), 500-502.

[17] Shanker, R., Fesshaye, H. and Yemane, A. (2015). On sizebiased Poisson-Lindley distribution and its applications to model thunderstorm. American Journal of Mathematics and Statistics, 5 (6), 354-360. 